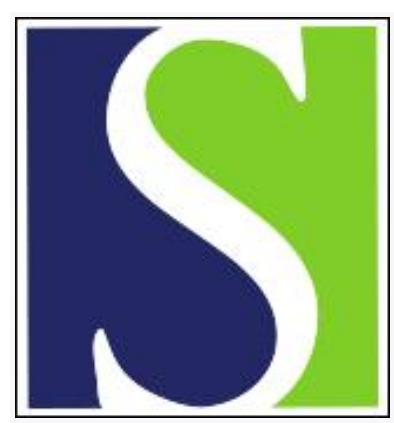

Scand J Work Environ Health 2000;26(6):459-460

https://doi.org/10.5271/sjweh.568

Issue date: Dec 2000

Modification of lung cancer prevention by gene-nutrient interaction

by Vainio $\mathrm{H}$

This article in PubMed: www.ncbi.nlm.nih.gov/pubmed/11201391 


\section{Modification of lung cancer prevention by gene-nutrient interaction}

Lung cancer is a major cause of death all over the world. Its link to tobacco use is well established. Of the over 4000 chemicals identified in tobacco smoke, there are at least 55 carcinogens, and 20 of them have induced lung tumors in at least one animal species (1). The strongest evidence exists for specific polyaromatic hydrocarbons, typified by benzo(a)pyrene (BaP), and tobacco-specific nitrosamines [eg, 4(methylnitrosamino)-1-(3-pyridyl)-1-butanone (NNK)].

Although nicotine addiction is the reason that people continue to smoke, nicotine itself is not considered to be carcinogenic. However, each cigarette contains a small dose of BaP, NNK, and other carcinogens. Even though each individual dose is small, the overall dose in years of smoking is considerable.

Most carcinogens in tobacco smoke require metabolic activation to exert their carcinogenic effects. There are also competing detoxification processes that are protective. The balance between metabolic activation ("phase I") and inactivation ("phase II") reactions is a target for chemopreventive agents. If metabolic activation can be decreased, or detoxification increased, then the ensuing toxicities should decrease.

A family of enzymes that is involved in phase $\mathrm{Il}$, by transforming lipid-soluble chemicals into watersoluble excretable conjugates, is the glutathione S-transferases (GST). Some of them, such as glutathione S-transferase M1 (GSTM1), are polymorphic to the extent that about $50 \%$ of the white population in Europe and North America has no GSTM1 enzymatic activity (ie, they carry null GSTM1 alleles, inactivated by a deletion of DNA coding sequences) (2). Because GST enzymes act on certain carcinogens in tobacco smoke and make them excretable, the possibility that the null GSTM1 genotype could confer heightened susceptibility to tobacco smoke is plausible. However, while associations between GSTM1 null genotypes and lung cancer risk have been observed in some studies, other studies have reported contrary findings, and the importance of this polymorphism in mediating the risk of smoking-related cancers remains unproved (3).

The concept of chemoprevention, the use of chemical agents to inhibit and reverse lung carcinogenesis, has great appeal, especially among those who have stopped smoking but continue to have a high risk of developing cancer because of past exposure to carcinogens in smoke. Isothiocyanates form a group of naturally occurring chemicals in cruciferous vegetables - broccoli, cauliflower, cabbage, watercress, bok choy, among others - which have been shown experimentally to possess chemopreventive activity against various cancers, including NNK-induced pulmonary carcinogenesis (4). Isothiocyanates can profoundly affect carcinogen metabolism. According to measurements of urinary metabolites, the consumption of watercress, a rich source of isothiocyanates, by smokers alters the profile of NNK metabolism (5). Isothiocyanates are conjugated by GST to excretable conjugates; while they are substrates for GST, they can also induce GST. There are also interindividual differences in the metabolism of chemopreventive isothiocyanates. A recent study suggests that people who are deficient in GSTM1 and who consume broccoli are protected against colon cancer because of a less efficient metabolism of isothiocyanates $(6,7)$.

Isothiocyanates were recently reported to provide protection against lung cancer in a nested casereferent study within a cohort of 18244 men, 45-64 years of age, in Shanghai, China (8). This study demonstrated a unique gene-nutrient interaction. Subjects genetically deficient in the GSTM1 enzyme 
(which eliminates isothiocyanates from the body) benefitted the most from cruciferous vegetables, presumably because isothiocyanates stayed around longer to confer their protective effect. Among all the subjects, those with detectable levels of isothiocyanates in their urine had a $40 \%$ decreased risk of lung cancer. Among the subjects lacking the GSTM1 enzyme, isothiocyanate intake was associated with a $64 \%$ decrease in the risk of lung cancer. Conversely, people with homozygous deletion of GSTM1 were at increased risk of lung cancer when they had no intake of protecting isothiocyanates.

The report of London et al (8) represents an important step towards elucidating the modifying role of GSTM1 polymorphism in lung-cancer risk, and it suggests that the previously published conflicting results between GSTM1 genotypes and smoking-related cancers could be due to variations in diet between populations. It is an illustrative example of the complexities we are facing when trying to understand the role of genetic factors in disease causation. Such a role can only be discovered by innovative epidemiologic approaches, with prospective sample collections as in the study by London et al (8). Measurements of both human uptake and differences in the metabolism of putative chemopreventive agents are necessary if the potential anticarcinogenic action is to be evaluated.

\title{
References
}

1. Hecht SS. Tobacco smoke carcinogens and lung cancer. JNCI 1999;91:1194-210.

2. Seidegard J, Vorachek WR, Pero RW, Pearson WR. Hereditary differences in the expression of human glutathione Stransferase activity on trans-stilbene oxide are due to a gene deletion. Proc Natl Acad Sci USA 1988;85:7293-97

3. Strange RC, Fryer AA. The glutathione S-transferases: influence of polymorphism on cancer susceptibility. In: Vineis $P$, Malats N, Lang M, d'Errico A, Caporaso N, Cusick J, et al, editors. Metabolic polymorphisms and susceptibility to cancer. Lyon: International Agency for Research on Cancer (IARC) 1999:231-49. IARC scientific publications, no 148.

4. Hecht SS. Anticarcinogenesis by isothiocyanates, indole-3-carbinol, and allium thiols. In: Eisenbrand G, Dyan AD, Elias PS, Grunow W, Schlatter J, editors. Carcinogenic and anticarcinogenic factors in food, Weinheim: Deutsche Forschungsgemeinschaft, Wiley-VCH, 2000:306-33.

5. Carmella SG, Borukhova A, Akerkar SA, Hecht SS. Analysis of human urine for pyridine-N-oxide metabolites of 4(methylnitrosamino)-1-(3-pyridyl)-1-butanone, a tobacco-specific lung carcinogen. Cancer Epidemiol Biomarkers Prev 1997;6:113-20.

6. Lin HJ, Probst-Hensch NM, Louie AD, Kan IH, Witte JS, Ingles SA, et al. Glutathione transferase null genotype, broccoli, and lower prevalence of colorectal adenomas. Cancer Epidemiol Biomarkers Prev 1998;7:647_52.

7. Ketterer, B. Dietary isothiocyanates as confounding factors in the molecular epidemiology of colon cancer. Cancer Epidemiol Biomarkers Prev 1998;7:645-6.

8. London SJ, Yuan J-M, Chung F-L, Gao Y-T, Coetzee GA, Ross RK, et al. Isothiocyanates, glutathione S-transferase M1 and T1 polymorphisms, and lung-cancer risk: a prospective study of men in Shanghai, China. Lancet 2000;356:724-9.

\author{
Harri Vainio \\ Unit of Chemoprevention \\ International Agency for Research on Cancer \\ 150 Cours Albert Thomas \\ F-69372 Lyon, Cedex 08, France
}

